\title{
Como tratar os dados da amostra do Censo Demográfico 2000 na obtenção de estimativas para os "indígenas"? Um estudo a partir das Terras Indígenas Xavante, Mato Grosso
}

\author{
Nilza de Oliveira Martins Pereira* \\ José André Moura de Brito** \\ Sonia Albieri*** \\ Antonio José Ribeiro Dias**** \\ Ricardo Ventura Santos $* \star \star \star *$
}

\begin{abstract}
A demografia dos povos indígenas no Brasil é ainda muito pouco conhecida nos seus mais diversos aspectos. Nos últimos anos, vem acontecendo uma ampliação de interesse pelo tema, com a publicação de diversos estudos sobre demografia dos "indígenas" com base nos dados censitários. Uma dificuldade metodológica importante diz respeito à expansão da amostra dos censos, já que as terras indígenas não foram definidas originalmente como áreas de ponderação. Este trabalho apresenta estimativas para as variáveis do questionário da "amostra", considerando um conjunto de setores censitários pertencentes a terras indígenas e utilizando como estudo de caso as Terras Xavante localizadas no leste de Mato Grosso, constituídas por seis áreas não-contíguas. Trata-se de um exercício metodológico que visa comparar e avaliar as estimativas produzidas segundo os pesos gerados na época de divulgação do Censo 2000 e os novos pesos calculados a partir de metodologia aqui apresentada. Do ponto de vista metodológico, esse procedimento é inovador, pois pode ser útil para estimar, com base nos dados da amostra do Censo 2000, características de áreas nãocontíguas e diferentes daquelas definidas para a expansão da amostra como originalmente realizada e divulgada pelo IBGE.
\end{abstract}

Palavras-chave: Censos demográficos. Cor ou raça. Indígenas. Amostragem.

\section{Introdução}

A demografia dos povos indígenas no Brasil é ainda muito pouco conhecida nos seus mais diversos aspectos, incluindo volume populacional, composição por sexo e idade, perfil de natalidade, mortalidade e migração, aspectos socioeconômicos (educação e trabalho), entre outros (MCSWEENEY; ARPS, 2005; PAGLIARO et al., 2005). Nos últimos anos, vem acontecendo uma ampliação de interesse pelo tema, com a publicação de diversos estudos, seja com a análise baseada em estudos de casos específicos realizados em comunidades indígenas em diversas regiões do país (PAGLIARO et al., 2005), seja por meio de análises de dados censitários (IBGE, 2005). Sobre os dados censitários, têm sido publicados diversos estudos nos últimos anos (AZEVEDO, 2000;

\footnotetext{
* Estatística - Diretoria de Pesquisas do Instituto Brasileiro de Geografia e Estatística (IBGE).

** Estatístico - Coordenação de Qualidade e Métodos do Instituto Brasileiro de Geografia e Estatística (IBGE).

*** Estatística - Coordenação de Qualidade e Métodos do Instituto Brasileiro de Geografia e Estatística (IBGE).

**** Estatístico - Coordenação de Qualidade e Métodos do Instituto Brasileiro de Geografia e Estatística (IBGE).

${ }^{* * * * *}$ Antropólogo - Escola Nacional de Saúde Pública, Fundação Oswaldo Cruz (Fiocruz) e Departamento de Antropologia, Museu Nacional, Universidade Federal do Rio de Janeiro (UFRJ).
} 
IBGE, 2005; KENNEDY et al., 2000; PEREIRA et al., 2005, 2009; PERZ et al., 2008).

$\mathrm{Na}$ coleta dos dados nos Censos Demográficos 1991 e 2000, foram usados dois modelos de questionário: um básico, aplicado nas unidades não selecionadas para a amostra e contendo perguntas referentes às características que foram investigadas para toda a população; e outro aplicado somente nos domicílios selecionados para a amostra. Neste caso, além das perguntas que também constam do questionário básico, havia outras mais detalhadas sobre características do domicílio e de seus moradores, referentes aos temas religião, cor ou raça, deficiência, migração, escolaridade, fecundidade, nupcialidade, mão de obra e rendimento (IBGE, 2003; ALBIERI, 2008).

A técnica de amostragem aplicada aos censos brasileiros mais recentes data de 1960 (IBGE, 2003). O desenho amostral adotado compreende a seleção sistemática e com equiprobabilidade, dentro de cada setor censitário, ${ }^{1}$ de uma amostra dos domicílios particulares e das famílias ou componentes de grupos conviventes recenseados em domicílios coletivos, com fração amostral constante para setores de um mesmo município. No que concerne ao Censo 2000, foram definidas duas frações amostrais distintas: $10 \%$ para os municípios com população estimada superior a 15.000 habitantes; e $20 \%$ para os demais municípios. Essa metodologia foi aplicada de forma semelhante ao Censo Demográfico de 1991 (IBGE, 2003; SILVA; BIANCHINI; ALBIERI, 1993).

Tanto no Censo de 1991 quanto no de 2000, a investigação dos "indígenas" foi feita com base no quesito "cor ou raça", presente no questionário da amostra. $\mathrm{Na}$ prática, como se trata de uma amostra, ocorre que os dados sobre os "indígenas", ou qualquer outra categoria de "cor ou raça" (branca, parda, preta e amarela), precisam ser, estatisticamente falando, expandidos, de modo a se produzirem estimativas para a população total. Todavia, mesmo no caso da amostra do censo, dimensionada de forma a propiciar medidas de precisão adequadas para níveis geográficos variados, a produção de estimativas associadas a determinados segmentos populacionais sociodemograficamente diferenciados em um contexto espacial mais desagregado pode ser inviável, sob a pena de uma perda de precisão. Ou seja, o erro padrão associado a tais estimativas pode ser muito grande e, consequentemente, tais estimativas não seriam confiáveis.

No Brasil, existem aproximadamente 470 Terras Indígenas oficialmente reconhecidas pela Fundação Nacional do Índio (Funai), que correspondem a cerca de 15\% da extensão do território nacional e abrigam povos indígenas de cerca de 220 etnias. Por razões técnicas, os setores censitários localizados nas Terras Indígenas não são facilmente identificáveis na base de dados dos Censos Demográficos (ver PEREIRA et al., 2009), o que dificulta a realização de análises sobre a categoria "indígena" com base no quesito "cor ou raça". Um elemento complicador adicional é a pequena proporção desse segmento populacional, que não atingiu $0,5 \%$ da população total do Brasil, segundo o Censo Demográfico 2000 (IBGE, 2005).

Este trabalho tem por objetivo apresentar estimativas para as variáveis do questionário da "amostra", considerando um conjunto de setores censitários pertencentes às terras indígenas e utilizando como estudo de caso as Terras Xavante localizadas no leste de Mato Grosso. Essas terras são constituídas por seis áreas não-contíguas, originalmente não definidas como área de ponderação no Censo 2000. Trata-se de um exercício metodológico que visa comparar e avaliar as estimativas produzidas segundo os pesos gerados na época de divulgação do Censo 2000 e novos pesos calculados para o conjunto das Terras Indígenas Xavante a partir da metodologia aqui apresentada. A avaliação de tais estimativas será efetuada com base no cálculo dos seus coeficientes de variação. Do ponto

\footnotetext{
1 Setor censitário é a unidade de controle cadastral formada por área contínua urbana ou rural, cuja dimensão e número de domicílios ou de unidades não-residenciais permitem ao recenseador cumprir suas atividades censitárias em um prazo determinado, respeitando o cronograma de atividades (IBGE, 2000).
} 
de vista metodológico, esse procedimento é inovador, pois pode ser útil para estimar, com base nos dados da amostra do Censo 2000, características de áreas não-contíguas e diferentes daquelas definidas para a expansão da amostra como originalmente realizada e divulgada pelo IBGE.

\section{Fontes de dados e metodologia}

Para o Censo 2000, foram usados métodos e sistemas automáticos de formação de áreas de ponderação, que conjugam critérios tais como tamanho (para permitir estimativas com qualidade estatística em áreas pequenas), contiguidade (no sentido de serem constituídas por setores vizinhos e contínuos em um dado espaço geográfico) e homogeneidade em relação a um conjunto de características populacionais e de infraestrutura conhecidas (IBGE, 2003). Essas áreas de ponderação, a partir das quais se procede a expansão da amostra, são definidas como unidades geográficas formadas por agrupamentos mutuamente exclusivos de setores censitários. Para as áreas de ponderação, são aplicados os procedimentos de calibração das estimativas com as informações conhecidas para o total da população.

Para fins do Censo 2000, foram definidas, para todo o Brasil, 9.336 áreas de ponderação e, tal como nos censos anteriores, a metodologia de expansão da amostra foi aplicada independentemente para cada uma delas (IBGE, 2003). O tamanho dessas áreas, em termos de número de domicílios e de população, não pode ser muito reduzido, sob pena de perda de precisão de suas estimativas. As áreas de ponderação foram definidas considerando esta condição e, também, os níveis geográficos mais detalhados da base operacional do censo demográfico, como forma de atender a demandas por informações em níveis geográficos menores que os municípios (IBGE, 2003).

A área analisada neste trabalho corresponde a um conjunto de seis Terras Indígenas Xavante do Estado do Mato Grosso (Areões, Marechal Rondon, Parabubure, Pimentel Barbosa, Sangradouro - Volta Grande e São Marcos) (Figura 1). Não foi

FIGURA 1

Localização das Terras Indígenas Xavante, Mato Grosso

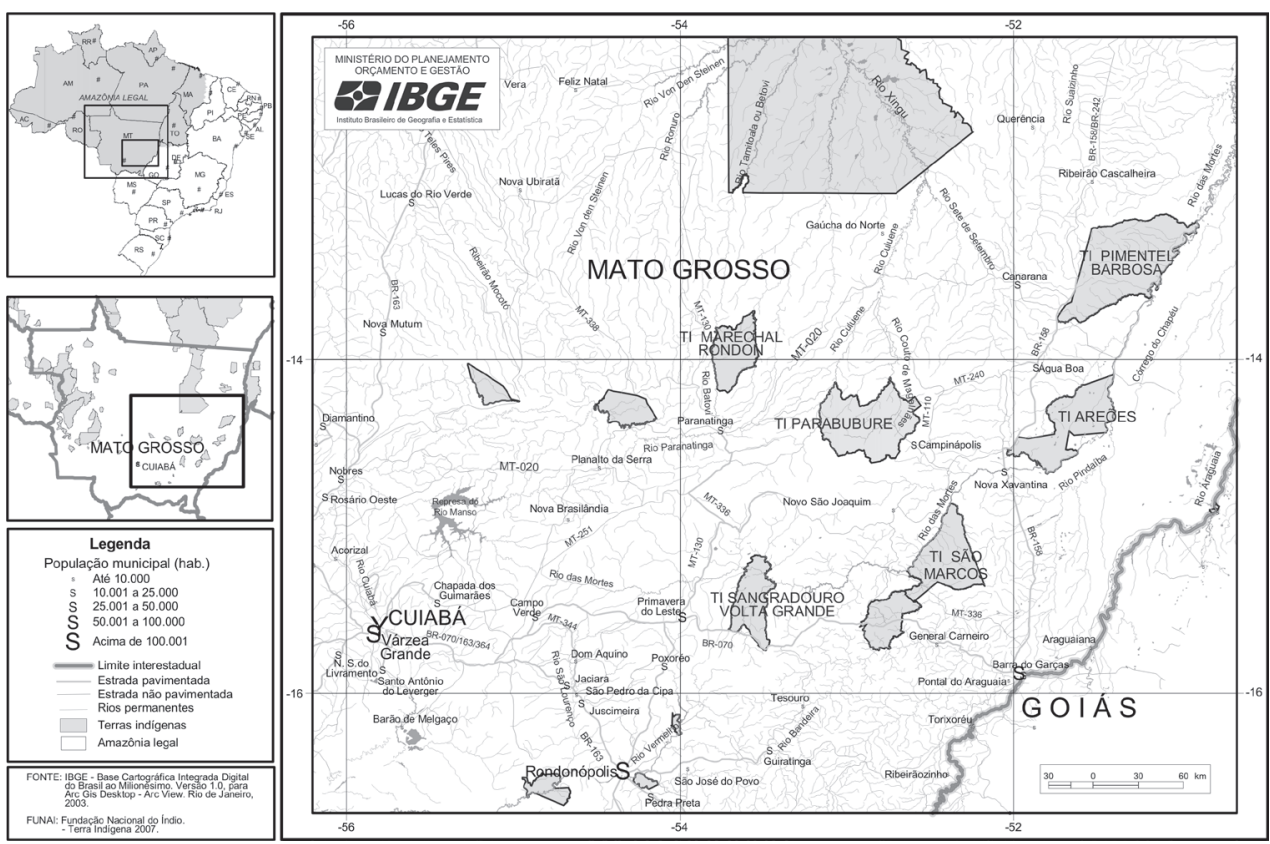

Fonte.: Elaborado pela Diretoria de Geociências - Coordenação de Geografia - IBGE. 
incluída a Terra Indígena Maraiwatsede, já que sua ocupação pelos Xavante aconteceu depois de 2000. As Terras Xavante não constituem áreas contíguas; portanto, não contemplam os critérios que definem uma área de ponderação (ver SILVA et al., 2002).

Nas seis Terras Xavante foram localizados 64 setores censitários na base do Censo 2000, distribuídos da seguinte forma: nove em Areões; um em Marechal Rondon; 41 em Parabubure; cinco em Pimentel Barbosa; cinco em Sangradouro - Volta Grande; e três em São Marcos. ${ }^{2}$ No âmbito do presente trabalho, foi conduzida uma expansão da amostra especificamente para essa área (ou seja, o conjunto dos 64 setores), calculandose novos pesos.

O método para obtenção dos novos pesos foi o mesmo empregado no Censo 2000, utilizando-se um processo de calibração (DIAS; ALBIERI, 2004) que buscou ajustar os pesos iniciais (inverso da fração amostral de domicílios), de maneira que, no conjunto de setores censitários das Terras Indígenas Xavante, agora definindo uma nova área de ponderação, ao se aplicarem os pesos calibrados às variáveis auxiliares, fossem obtidos os totais já conhecidos para todas as unidades da população que constituem o universo da pesquisa. Dessa maneira, além de buscar a melhoria da precisão das estimativas, procuram-se estimativas mais consistentes para as variáveis pesquisadas somente pelo questionário da amostra (IBGE, 2003).

Tendo em vista que se deseja produzir um conjunto de estimativas com base nos dados da população indígena contidos no questionário da amostra do Censo 2000, devem-se utilizar pesos para cada um dos domicílios indígenas pesquisados. Esses pesos são atribuídos ao próprio domicílio e a cada um de seus moradores. O método utilizado para obtenção dos pesos foi um processo de calibração em relação a um conjunto de variáveis auxiliares ${ }^{3}$ (restrições), para as quais se conhecem os totais populacionais, já que tais variáveis auxiliares foram levantadas pelo questionário básico (IBGE, 2003). Assim, o conjunto das Terras Xavante foi considerado uma área de ponderação, de aproximadamente 10 mil habitantes, aplicando-se a metodologia empregada para obtenção dos pesos originais utilizados na divulgação dos resultados do Censo 2000.

A metodologia para o cálculo dos pesos calibrados foi baseada no método dos Mínimos Quadrados Generalizados (MQG) (KARIYA; KURATA, 2004), que corresponde a uma técnica de otimização matemática amplamente utilizada nas áreas de estatística e economia, entre outras, e visa encontrar o melhor ajustamento para um conjunto de dados, tentando minimizar a soma dos quadrados das diferenças entre a curva ajustada e os dados (tais diferenças são chamadas resíduos). Um requisito é que os erros em cada medida sejam distribuídos aleatoriamente, com função densidade gaussiana, e que os resíduos sejam independentes.

Para o cálculo dos pesos do censo, na aplicação do método foi definido um conjunto de variáveis auxiliares referentes a características de domicílios ou de pessoas, que constituem um subconjunto das variáveis comuns à amostra e ao universo e definem as restrições associadas ao modelo que será resolvido de forma a determinar os pesos.

\footnotetext{
2 Entre os 64 setores localizados nas terras Xavante, 54 (88,5\%) foram classificados como "setor de aldeia indígena" por parte do IBGE. Considerando-se que, no Estado de Mato Grosso, 146 setores foram classificados como "de aldeia indígena", deduz-se que a malha dos setores censitários para o caso Xavante foi particularmente bem construída e classificada (ver também PEREIRA et al., 2009).

${ }^{3}$ As restrições (que constituem o conjunto 1 de restrições para calibração) inicialmente definidas para a aplicação da metodologia MQG, para cada uma das áreas de ponderação, são as seguintes:

- em unidades domiciliares (domicílios particulares ocupados + famílias ou pessoas sós em domicílios coletivos): total de pessoas; total de unidades domiciliares; total de pessoas e por grupos de idade quinquenais de 0 a 4 anos até 60 a 69 anos e 70 anos ou mais; número de pessoas do sexo masculino e por grupos de idade quinquenais de 0 a 4 anos até 50 a 59 anos e 60 anos ou mais; número de pessoas moradoras na área urbana; número de pessoas do sexo feminino moradoras na área urbana e na rural;

- em domicílios particulares permanentes ocupados: número de pessoas do sexo masculino que são chefes ou individuais; total de pessoas; total de domicílios e domicílios urbanos; número de domicílios com 1 ou 2 moradores; com 3 moradores; 4 moradores; com 5 moradores; e com 6 ou mais moradores.
} 
Foram impostos limites nos pesos finais para evitar pesos muito pequenos ou muito grandes. O limite mínimo utilizado foi 1 , de maneira que um domicílio representasse pelo menos ele próprio, e o máximo foi definido como cinco vezes o peso médio esperado: 25 no caso de áreas de ponderação associadas a municípios grandes (mais de 15.000 habitantes e fração amostral planejada de $20 \%$ ), com peso médio esperado de 5; e 50 para áreas de ponderação associadas aos municípios pequenos (até 15.000 habitantes e fração amostral planejada de $10 \%$ ), com peso médio esperado de 10. Sem a utilização desses limites, o método MQG pode gerar pesos negativos ou muito grandes, o que não teria sentido prático.

Uma vez definidos o modelo, as restrições associadas e os limites inferior e superior para os pesos finais (peso inicial $x$ fator de ajuste), a aplicação da metodologia do MQG envolveu o cálculo com matrizes, inclusive inversão. Por esta razão, as restrições definidas, que por sua vez dão origem a uma dessas matrizes, devem satisfazer algumas condições essenciais, sendo a principal a de não serem linearmente dependentes (redundantes). Também foi considerado o conceito de restrições quase-linearmente dependentes (e, portanto, quase redundantes), que afetam a estabilidade da solução do modelo. Outras duas condições impostas para a aplicação dessa metodologia referem-se à sua significância estatística. $\mathrm{O}$ tamanho da restrição, medido como o número de domicílios aos quais a restrição se aplica em uma dada área de ponderação, não deve ser muito pequeno, sob pena de tornar instável o processo de estimação. Se uma restrição não atinge um número mínimo de unidades domiciliares, fixado em função da fração de amostragem, é considerada rara.

Além disso, uma restrição definida pode causar a obtenção de um peso muito grande ou muito pequeno, quando comparado com o peso médio esperado em função da fração amostral adotada na área de ponderação, ou até um peso negativo, constituindo-se em restrição geradora de peso extremo.

Dessa forma, o programa de ajuste do modelo incorpora procedimentos de eliminação de restrições que se enquadrem nas condições anteriores, observando a ordem que se segue: restrições raras, restrições redundantes, restrições quase redundantes e restrições responsáveis por pesos extremos.

O método MQG adotado baseou-se em proposta de Bankier (1990) e, para sua implementação, um sistema em linguagem SAS foi desenvolvido por técnicos do IBGE. O produto final da aplicação dessa metodologia é um peso ajustado para cada unidade domiciliar da amostra, ou seja, cada um dos questionários da amostra, que é repetido nos registros de cada pessoa moradora na unidade domiciliar (DIAS; ALBIERI, 2004; IBGE, 2003).

Estimativas provenientes de pesquisas realizadas por amostragem têm uma variabilidade que é inerente ao processo de amostragem. Assim, a avaliação dos chamados erros amostrais é um ponto fundamental, pois dela decorre o grau de confiança nas conclusões analíticas que subsidiam a tomada de decisão. Para cada estimativa derivada da pesquisa é possível obter uma medida de precisão que auxilia na análise e interpretação dos dados (ALBIERI; PEREIRA; BRITO, 2006).

Para o presente trabalho, com a finalidade de verificar o grau de precisão das estimativas, foram calculados os erros amostrais estimados da população autodeclarada indígena no Censo 2000, para os setores censitários localizados nas Terras Indígenas Xavante, utilizando os dados investigados nos questionários da amostra.

Para os cálculos dos erros amostrais e dos seus respectivos coeficientes de variação (CV), levou-se em conta o esquema de amostragem estratificada, que é o desenho do censo. Tais cálculos foram efetuados a partir da utilização de um conjunto de macros implementadas em linguagem SAS e do software GES (Generalized Estimation System). Considerando-se, por exemplo, o cálculo do estimador de variância associado a uma estimativa de total no presente esquema de amostragem, utilizou-se a informação dos pesos finais $w$ obtidos para a expansão da amostra $(w=d . g)$ e que foram aplicados para cada um dos domicílios pesquisados na amostra e cada um de seus moradores. $O$ valor $d$ representa o peso inicial do desenho 
amostral, que é o inverso da fração amostral usada para a seleção da amostra, e o valor $g$ representa o fator de ajuste, obtido durante o processo de calibração de cada área de ponderação (domínio de calibração) do Censo Demográfico 2000. Com base no trabalho de Hidiroglou (1991), a expressão analítica do estimador da variância para um total estimado $\hat{Y}$ pode ser escrita da seguinte forma:

$$
\begin{aligned}
& v(\hat{Y})=\sum_{h=1}^{L}\left(1-\frac{n_{h}}{N_{h}}\right) \frac{n_{h}}{n_{h}-1} \sum_{i=1}^{n_{h}}\left(b_{h i}-\bar{b}_{h}\right)^{2} \\
& \text { sendo } b_{h i}=d_{h i} g_{h i} e_{h i} \\
& \text { onde: }
\end{aligned}
$$

$d_{h i}$ é o peso do desenho, considerando o h-ésimo estrato e o i-ésimo conglomerado;

$g_{h i}$ é o fator de ajuste obtido no processo de calibração, considerando o $h$-ésimo estrato e o i-ésimo conglomerado;

$e_{h i}$ é o resíduo obtido no processo de calibração, considerando a informação das variáveis auxiliares utilizadas nesse processo e o valor da variável $y$ para qual é feita a estimativa $\hat{Y}$, para o h-ésimo estrato e o i-ésimo conglomerado;

$L$ é o número de estratos, e $h$ representa o estrato;

$n_{h}$ é o número de elementos da amostra no h-ésimo estrato;

$N_{h}$ é o número de elementos da população no h-ésimo estrato.

Pode-se definir o coeficiente de variação (CV) de uma estimativa $\hat{Y}$ de acordo com a seguinte expressão:

$$
c v(\hat{Y})=\sqrt{v(\hat{Y})} / \hat{Y}
$$

Conforme mencionado anteriormente, ao se efetuar o cálculo da variância, considerando o plano amostral estratificado do Censo 2000, cada estrato $h$ corresponde a um setor e cada conglomerado $i$ refere-se a um domicílio.

Os valores de erros-padrão e dos seus respectivos coeficientes de variação foram calculados para um conjunto de estimativas associadas às características da população indígena Xavante.

Além do cálculo dos coeficientes de variação, foi calculada uma medida de eficiência relativa definida pela seguinte expressão:

$$
\operatorname{eff}(\hat{Y})=\frac{v(\hat{Y})_{n v}}{v(\hat{Y})_{p c}}
$$

onde $v(\hat{Y})_{n v}$ e $v(\hat{Y})_{p c}$ são, respectivamente, as variâncias de uma estimativa $\hat{Y}$, considerando-se os novos pesos obtidos para os domicílios que compõem os 64 setores censitários das terras Xavante e os pesos originais do Censo 2000. Os valores de eff inferiores a 1 indicam um ganho de precisão ao se adotarem os novos pesos, enquanto os valores superiores a 1 indicam uma perda de precisão.

A variável selecionada para avaliar o grau de precisão das estimativas da população residente nas Terras Indígenas Xavante foi a "cor ou raça". Especificamente para a população que se autodeclarou "indígena" no conjunto dos setores censitários, foram analisadas as variáveis relacionadas aos seguintes temas: sexo; idade; religião; migração; nupcialidade; educação; e trabaIho. Verificou-se também o quantitativo de pessoas que forneceram as suas próprias informações.

\section{Resultados e discussão}

Analisando as Tabelas 1 a 3, observase que, em geral, não há grandes perdas ou ganhos de precisão ao se compararem as estimativas derivadas da aplicação dos novos pesos em relação àquelas que empregaram os pesos originais do Censo 2000. Esta conclusão deriva do fato de que a maior parte das estimativas produzidas a partir da utilização dos dois conjuntos de pesos tem coeficientes de variação (CVs) próximos. Assim, utilizando os pesos originais do Censo 2000, grande parte das estimativas produzidas para a população indígena Xavante $(61,3 \%)$ tem CVs de até $15 \%$, alcançando $68,9 \%$ para as estimativas cujos respectivos CVs são de até $20 \%$.

Considerando-se os novos pesos, foi observado um percentual de $60,4 \%$ em relação às estimativas que tiveram $\mathrm{CVs}$ de até $15 \%$, ou seja, ligeiramente inferior ao percentual referente aos pesos originais. Finalmente, no caso das estimativas com CVs de até $20 \%$, verificou-se o mesmo 
percentual dos pesos originais. O plano amostral definido para o censo tenta garantir que em todos os municípios as estimativas sejam obtidas com níveis de precisão equivalentes, utilizando uma classificação na qual são consideradas estimativas com precisão razoável (aceitável) aquelas cujos CVs sejam inferiores a 15\% (ALBIERI, 1999).

As informações da estrutura por sexo e idade provenientes do universo (os setores censitários das Terras Xavante), quando comparadas com os resultados da amostra expandida (também para as Terras Xavante), segundo os pesos originais, já demonstravam estruturas bem semelhantes. Convém esclarecer que a aplicação das frações de amostragem nos diversos municípios fez com que a dimensão da amostra do censo resultasse robusta o suficiente, propiciando medidas de precisão adequadas para níveis geográficos variados, como forma de atender às demandas por informações municipais e, dependendo da dimensão populacional do município, até mesmo para áreas menores (Tabela 4). Desse modo, quando se utilizaram os novos pesos, já era de se esperar que não existiriam grandes diferenças entre as estimativas, com os resultados apresentando convergência. Tal proximidade pode ser justificada pela não existência de grandes perdas ou ganhos de precisão, ao se compararem as estimativas derivadas da aplicação dos novos pesos em relação àquelas que empregaram os pesos originais do Censo 2000.

Para o conjunto de variáveis analisadas, observou-se um equilíbrio entre os valores da eficiência, isto é, ora para ganho ora para perda de precisão. É fundamental frisar que as estimativas com os novos pesos respeitam um pressuposto de grande importância: as Terras Xavante foram consideradas uma área de ponderação, o que não era o caso das estimativas derivadas da aplicação dos pesos originais.

Há outros aspectos referentes aos resultados que merecem consideração. Ainda que a maior parte das estimativas indicadas nas Tabelas 1 a 3 tenha CVs com valores aceitáveis, para diversas variáveis podem ser observados valores acima de $15 \%$. A obtenção de estimativas com precisão aceitável vale para características que não sejam muito raras, pois, quanto mais rara for a característica a se observar, mais difícil de estimar com qualidade. Pode ser observado

TABELA 1

Estimativas, coeficientes de variação e eficiência para pessoas residentes nas Terras Indígenas Xavante, a partir da utilização dos pesos originais e dos novos pesos, segundo sexo e cor ou raça Estado do Mato Grosso - 2000

\begin{tabular}{|c|c|c|c|c|c|}
\hline \multirow{2}{*}{ Sexo e cor ou raça } & \multicolumn{2}{|c|}{ Estimativas } & \multicolumn{2}{|c|}{ Coeficientes de variação (\%) } & \multirow{2}{*}{ Eficiência (\%) } \\
\hline & População (1) & População (2) & População (1) & População (2) & \\
\hline Total & 9.605 & 9.644 & 0,00 & 0,00 & - \\
\hline Branca & 10 & 8 & 91,55 & 92,41 & 1,5 \\
\hline Parda & 22 & 20 & 82,00 & 81,44 & 1,2 \\
\hline Indígena & 9.500 & 9.540 & 0,27 & 0,26 & 1,1 \\
\hline Ignorada & 74 & 76 & 23,20 & 23,59 & 0,9 \\
\hline Homens & 4.911 & 5.093 & 0,00 & 0,00 & - \\
\hline Branca & 0 & 0 & - & - & - \\
\hline Parda & 11 & 10 & 82,00 & 81,44 & 1,1 \\
\hline Indígena & 4.866 & 5.041 & 0,28 & 0,30 & 0,8 \\
\hline Ignorada & 34 & 42 & 34,39 & 33,03 & 0,7 \\
\hline Mulheres & 4.694 & 4.551 & 0,00 & 0,00 & - \\
\hline Branca & 10 & 8 & 91,55 & 92,41 & 1,5 \\
\hline Parda & 11 & 10 & 82,00 & 81,44 & 1,1 \\
\hline Indígena & 4.634 & 4.499 & 0,39 & 0,36 & 1,2 \\
\hline Ignorada & 39 & 34 & 31,78 & 34,79 & 1,1 \\
\hline
\end{tabular}

Fonte: IBGE. Censo Demográfico 2000. Tabulação especial.

(1) População gerada com novos pesos para o conjunto das Terras Indígenas.

(2) População gerada com os pesos originais. 
TABELA 2

Estimativas, coeficientes de variação e eficiência para a população autodeclarada indígena residente nas Terras Indígenas Xavante, a partir da utilização dos pesos originais e dos novos pesos, por sexo, segundo grupos de idade Estado do Mato Grosso - 2000

\begin{tabular}{|c|c|c|c|c|c|c|}
\hline \multirow{2}{*}{$\begin{array}{l}\text { Grupos de idade } \\
\text { (em anos) }\end{array}$} & \multicolumn{2}{|c|}{ Total } & \multicolumn{2}{|c|}{ Homens } & \multicolumn{2}{|c|}{ Mulheres } \\
\hline & $\begin{array}{c}\text { População } \\
\text { (1) }\end{array}$ & $\begin{array}{c}\text { População } \\
\text { (2) }\end{array}$ & $\begin{array}{c}\text { População } \\
\text { (1) }\end{array}$ & $\begin{array}{c}\text { População } \\
\text { (2) }\end{array}$ & $\begin{array}{c}\text { População } \\
\text { (1) }\end{array}$ & $\begin{array}{c}\text { População } \\
\text { (2) }\end{array}$ \\
\hline \multicolumn{7}{|l|}{ Estimativas } \\
\hline Total & 9.500 & 9.540 & 4.866 & 5.041 & 4.634 & 4.499 \\
\hline 0 a 4 & 2.039 & 2.035 & 1.022 & 1.042 & 1.017 & 993 \\
\hline 5 a 9 & 1.641 & 1.557 & 823 & 824 & 818 & 734 \\
\hline 10 a 14 & 1.305 & 1.285 & 620 & 660 & 686 & 625 \\
\hline 15 a 19 & 1.082 & 1.134 & 577 & 625 & 505 & 509 \\
\hline 20 a 24 & 820 & 891 & 426 & 496 & 394 & 395 \\
\hline 25 a 29 & 675 & 611 & 360 & 306 & 315 & 305 \\
\hline 30 a 34 & 490 & 500 & 238 & 254 & 252 & 246 \\
\hline 35 a 39 & 337 & 390 & 178 & 176 & 159 & 214 \\
\hline 40 a 44 & 149 & 144 & 89 & 96 & 60 & 48 \\
\hline 45 a 49 & 88 & 53 & 56 & 30 & 32 & 23 \\
\hline 50 a 54 & 91 & 152 & 61 & 103 & 30 & 48 \\
\hline 55 a 59 & 47 & 64 & 19 & 23 & 28 & 41 \\
\hline 60 a 64 & 85 & 71 & 34 & 22 & 51 & 49 \\
\hline 65 a 69 & 137 & 155 & 81 & 108 & 56 & 47 \\
\hline 70 a 74 & 131 & 144 & 73 & 85 & 58 & 59 \\
\hline 75 a 79 & 160 & 138 & 83 & 66 & 78 & 72 \\
\hline 80 ou mais & 222 & 216 & 127 & 126 & 95 & 90 \\
\hline \multicolumn{7}{|c|}{ Coeficientes de variação (\%) } \\
\hline Total & 0,30 & 0,26 & 0,28 & 0,30 & 0,39 & 0,36 \\
\hline 0 a 4 & 0,13 & 0,13 & 0,25 & 0,25 & 0,00 & 0,00 \\
\hline 5 a 9 & 0,48 & 0,54 & 0,76 & 0,73 & 0,59 & 0,80 \\
\hline 10 a 14 & 0,71 & 0,86 & 5,83 & 5,23 & 5,40 & 5,64 \\
\hline 15 a 19 & 0,00 & 0,00 & 6,72 & 6,39 & 7,68 & 7,84 \\
\hline 20 a 24 & 0,45 & 0,40 & 6,37 & 5,99 & 6,87 & 7,52 \\
\hline 25 a 29 & 2,88 & 2,88 & 2,49 & 2,76 & 3,84 & 3,43 \\
\hline 30 a 34 & 0,34 & 0,31 & 0,69 & 0,61 & 0,00 & 0,00 \\
\hline 35 a 39 & 0,00 & 0,00 & 0,00 & 0,00 & 0,00 & 0,00 \\
\hline 40 a 44 & 0,00 & 0,00 & 0,00 & 0,00 & 0,00 & 0,00 \\
\hline 45 a 49 & 0,00 & 0,00 & 0,00 & 0,00 & 0,00 & 0,00 \\
\hline 50 a 54 & 11,55 & 9,36 & 11,69 & 9,85 & 28,23 & 26,44 \\
\hline 55 a 59 & 22,58 & 22,16 & 38,37 & 44,94 & 30,20 & 30,87 \\
\hline 60 a 64 & 21,02 & 31,58 & 33,92 & 61,32 & 25,99 & 29,26 \\
\hline 65 a 69 & 13,39 & 15,17 & 21,57 & 22,84 & 29,62 & 35,72 \\
\hline 70 a 74 & 18,47 & 18,75 & 24,39 & 23,20 & 32,53 & 32,58 \\
\hline 75 a 79 & 19,24 & 18,97 & 24,79 & 26,97 & 22,20 & 22,89 \\
\hline 80 ou mais & 17,02 & 15,30 & 18,99 & 16,62 & 23,23 & 21,78 \\
\hline \multicolumn{7}{|l|}{ Eficiência (\%) } \\
\hline Total & \multicolumn{2}{|c|}{1,4} & \multicolumn{2}{|l|}{0,8} & \multicolumn{2}{|c|}{1,2} \\
\hline 0 a 4 & \multicolumn{2}{|c|}{1,0} & \multicolumn{2}{|l|}{1,0} & \multicolumn{2}{|c|}{-} \\
\hline 5 a 9 & \multicolumn{2}{|c|}{0,9} & \multicolumn{2}{|l|}{1,1} & \multicolumn{2}{|c|}{0,7} \\
\hline 10 a 14 & 0,7 & & 1,1 & & & \\
\hline 15 a 19 & . & & 0,9 & & & \\
\hline 20 a 24 & 1,09 & & 0,8 & & & \\
\hline 25 a 29 & 1,22 & & 1,1 & & & \\
\hline 30 a 34 & 1,13 & & 1,1 & & & - \\
\hline 35 a 39 & - & & - & & & - \\
\hline 40 a 44 & - & & - & & & - \\
\hline 45 a 49 & - & & - & & & - \\
\hline 50 a 54 & 0,6 & & 0,5 & & & \\
\hline 55 a 59 & 0,6 & & 0,5 & & & \\
\hline 60 a 64 & 0,6 & & 0,7 & & & \\
\hline 65 a 69 & 0,6 & & 0,5 & & & \\
\hline 70 a 74 & 0,8 & & 0,8 & & & \\
\hline 75 a 79 & 1,4 & & 1,3 & & & \\
\hline 80 ou mais & 1,3 & & 1,3 & & & \\
\hline
\end{tabular}

Fonte: IBGE. Censo Demográfico 2000. Tabulação especial.

(1) População gerada com novos pesos para o conjunto das Terras Indígenas.

(2) População gerada com os pesos originais. 
TABELA 3

Estimativas, coeficientes de variação e eficiência para população autodeclarada indígena residente nas Terras Indígenas Xavante, a partir da utilização dos pesos originais e dos novos pesos, segundo características demográficas, sociais e econômicas Estado do Mato Grosso - 2000

\begin{tabular}{|c|c|c|c|c|c|}
\hline \multirow{2}{*}{$\begin{array}{l}\text { Características demográficas, } \\
\text { sociais e econômicas }\end{array}$} & \multicolumn{2}{|c|}{ Estimativas } & \multicolumn{2}{|c|}{ Coeficientes de variação (\%) } & \multirow{2}{*}{$\begin{array}{c}\text { Eficiência } \\
\text { (\%) }\end{array}$} \\
\hline & $\begin{array}{c}\text { População } \\
(1)\end{array}$ & População & $\begin{array}{l}\text { População } \\
\text { (1) }\end{array}$ & $\begin{array}{l}\text { População } \\
(2)\end{array}$ & \\
\hline Religião & 9.500 & 9.540 & 0,27 & 0,26 & 1,1 \\
\hline Católica apostólica romana & 5.353 & 5.592 & 3,90 & 3,63 & 1,1 \\
\hline Evangélica de missão & 205 & 216 & 24,18 & 24,32 & 0,9 \\
\hline Evangélica de origem pentecostal & 666 & 572 & 19,83 & 15,58 & 2,2 \\
\hline Outros evangélicos & 580 & 586 & 22,52 & 18,32 & 1,5 \\
\hline Espírita & 25 & 27 & 63,48 & 67,63 & 0,8 \\
\hline Outras religiosidades & 50 & 50 & 84,59 & 84,69 & 1,0 \\
\hline Sem religião & 2.590 & 2.449 & 6,02 & 6,41 & 1,0 \\
\hline Sem declaração & 31 & 49 & 45,91 & 49,21 & 0,4 \\
\hline Migração & 9.500 & 9.540 & 0,30 & 0,26 & 1,4 \\
\hline Nascidos na Terra Indígena & 8.779 & 8.826 & 0,83 & 0,84 & 0,9 \\
\hline Não nascidos na Terra Indígena & 721 & 714 & 9,21 & 9,39 & 1,0 \\
\hline No mesmo estado & 711 & 706 & 9,32 & 9,46 & 1,0 \\
\hline Goiás & 10 & 8 & 45,79 & 53,64 & 1,1 \\
\hline Nupcialidade & 5.820 & 5.948 & 0,40 & 0,38 & 1,1 \\
\hline Solteiros & 2.091 & 2.148 & 1,91 & 1,74 & 1,1 \\
\hline Que nunca viveu em união & 1.979 & 2.035 & 2,09 & 1,90 & 1,1 \\
\hline Que já viveu em união & 112 & 113 & 22,94 & 21,16 & 1,1 \\
\hline Casados & 3.572 & 3.671 & 1,12 & 1,07 & 1,0 \\
\hline Separados não judicialmente & 88 & 72 & 21,44 & 21,74 & 1,0 \\
\hline Desquitados ou divorciados & 22 & 12 & 43,29 & 52,05 & 0,8 \\
\hline Viúvos & 47 & 47 & 38,99 & 39,93 & 1,0 \\
\hline Taxa de alfabetização (\% por idade) & 66,3 & 67,2 & 1,90 & 1,82 & 1,1 \\
\hline 10 a 14 & 77,9 & 79,8 & 3,39 & 3,26 & 1,0 \\
\hline 15 a 19 & 81,2 & 81,5 & 3,42 & 3,31 & 1,1 \\
\hline 20 a 29 & 76,8 & 78,3 & 3,07 & 2,91 & 1,1 \\
\hline 30 a 39 & 66,9 & 66,5 & 5,71 & 5,91 & 0,9 \\
\hline 40 a 49 & 39,0 & 38,3 & 16,78 & 19,72 & 0,8 \\
\hline 50 a 59 & 42,4 & 48,8 & 16,92 & 12,59 & 1,4 \\
\hline 60 ou mais & 14,8 & 13,9 & 19,77 & 20,74 & 1,0 \\
\hline Condição de atividade e ocupação & 5.820 & 5.948 & 0,40 & 0,38 & 1,1 \\
\hline Economicamente ativos & 2.907 & 2.813 & 2,98 & 3,15 & 1,0 \\
\hline Ocupados & 2.891 & 2.799 & 2,97 & 3,13 & 1,0 \\
\hline Não ocupados & 16 & 15 & 59,13 & 65,63 & 0,9 \\
\hline Não economicamente ativos & 2.913 & 3.134 & 3,13 & 2,95 & 1,0 \\
\hline $\begin{array}{l}\text { Fornecimento das informações } \\
\text { do censo }\end{array}$ & 9.500 & 9.540 & 0,30 & 0,26 & 1,4 \\
\hline Foi o próprio informante & 411 & 425 & 10,11 & 9,10 & 1,2 \\
\hline Não foi o próprio informante & 9.089 & 9.116 & 0,48 & 0,49 & 1,0 \\
\hline
\end{tabular}

Fonte: IBGE. Censo Demográfico 2000. Tabulação especial.

(1) População gerada com novos pesos para o conjunto das Terras Indígenas.

(2) População gerada com os pesos originais.

TABELA 4

Terras Indígenas Xavante, segundo município de localização, população residente e fração amostral do município Estado do Mato Grosso - 2000

\begin{tabular}{lccc}
\hline Terras Indígenas Xavante & Municípios & $\begin{array}{c}\text { População } \\
\text { residente }\end{array}$ & $\begin{array}{c}\text { Fração amostral } \\
(\%)\end{array}$ \\
\hline Areões & Água Boa (atualmente está em Nova Nazaré) & 16.737 & 10,0 \\
Marechal Rondon & Paranatinga & 15.342 & 10,0 \\
Parabubure & Água Boa & 16.737 & 10,0 \\
& Campinápolis & 12.419 & 20,0 \\
São Marcos & Nova Xavantina & 17.832 & 10,0 \\
Pimentel Barbosa & Barra do Garças & 52.092 & 10,0 \\
& Ribeirão Cascalheira & 8.866 & 20,0 \\
Sangradouro & Canarana & 15.408 & 10,0 \\
& Novo São Joaquim & 9.464 & 20,0 \\
& Poxoréo & 20.030 & 10,0 \\
\hline
\end{tabular}

Fonte: IBGE. Malha municipal vigente na época do Censo Demográfico 2000. 
que, para a Terra Indígena Xavante, os CVs mais elevados são exatamente aqueles relacionados a eventos menos frequentes. É o caso, por exemplo, no número de indivíduos não indígenas ("brancos" e "pardos") residentes nas terras indígenas (Tabela 1). Também apresentam CVs superiores a 15\% as estimativas de tamanho de população nas faixas etárias mais elevadas (Tabela 2) e para diversas variáveis demográficas, sociais e econômicas (Tabela 3 ).

Este trabalho almeja, primordialmente, abordar os resultados derivados da aplicação de uma metodologia que visa realizar a expansão de características da amostra do Censo 2000 para terras indígenas não-contíguas, e não realizar a análise e interpretação dos dados do ponto de vista sociodemográfico. Não obstante, é importante sinalizar um aspecto de suma importância que pode ser derivado dos resultados aqui apresentados: em grande parte devido ao reduzido tamanho do número de observações, para muitas variáveis não é possível gerar estimativas confiáveis para a população residente nas Terras Xavante. É o caso da variável "cor ou raça" mencionada anteriormente. Por um lado, pode-se afirmar com segurança que nas Terras Xavante, na época do Censo 2000 , viviam aproximadamente 9.500 pessoas que se declararam "indígenas", mas, por outro, não é possível afirmar com confiabilidade os totais populacionais de autodeclarados "brancos" ou "pardos" (Tabela 1), já que os CVs associados a tais estimativas são muito elevados.

$\mathrm{Na}$ mesma linha, podem ser geradas estimativas quanto à distribuição segundo grupos etários de 5 a 49 anos, para homens e mulheres, já que os CVs estão sempre abaixo de 10\%-15\% (Tabela 2). Os CVs relativos às estimativas da população autodeclarada indígena por grupos de idade são substancialmente mais elevados para as faixas superiores a 50 anos. Neste caso, uma alternativa seria agregar esses grupos etários em faixas maiores (dez anos, por exemplo).

Observa-se, na Tabela 3, que para outras características demográficas há estimativas com CVs elevados, o que torna imperativo um cuidadoso exercício interpre- tativo a partir dos resultados. Desse modo, é possível gerar estimativas quanto ao número de pessoas que se declararam como "católicos apostólicos romanos" ou "sem religião"; contudo, os CVs associados às estimativas para as outras opções (evangélica de missão, evangélica de origem pentecostal, outros evangélicos e espíritas) são muito elevados (em geral acima de 20\%).

Deve-se enfatizar que esses problemas de estimação apontados, bastante evidentes no caso Xavante, não se referem unicamente aos "indígenas". Na prática, trata-se de uma questão presente, por exemplo, na análise de dados para um grande número de municípios no Brasil, em particular aqueles que contam com contingentes populacionais reduzidos. Para se dimensionar a questão, basta lembrar que, do total de 5.507 municípios investigados no Censo 2000, 64\% apresentavam população total de até $15 \mathrm{mil}$ habitantes. Em outras palavras, o elemento de cautela enfatizado anteriormente não é algo particular para as terras indígenas, mas se aplica para quaisquer situações, no âmbito dos censos nacionais, em que os eventos sejam pouco frequentes. É fundamental que todos aqueles envolvidos na análise dos dados censitários, seja trabalhando com "indígenas" ou não, estejam cientes desses problemas de estimação.

Como referido anteriormente, existem no Brasil cerca de 220 etnias indígenas, distribuídas em centenas de terras indígenas. Tal como os Xavante, há diversos povos cujas populações totalizam milhares de pessoas, distribuídas em duas, três ou mais terras indígenas não-contíguas. Este é o caso de povos como os Kayapó (Mato Grosso e Pará), Yanomami (Amazonas e Roraima) e Kaingang (Paraná, Santa Catarina e Rio Grande do Sul), somente para citar três exemplos. Tal como se procedeu neste trabalho, para essas e outras etnias, a partir da metodologia aqui apresentada, seria possível gerar estimativas para o conjunto das terras indígenas dos vários povos, possibilitando o delineamento de panoramas analíticos mais satisfatórios.

Com relação à replicação da metodologia aqui apresentada para os Xavante para outras terras indígenas no Brasil, é 
importante indicar certas especificidades. No caso Xavante, felizmente, constatou-se uma sobreposição entre os setores censitários referentes ao Censo 2000 e os limites das terras indígenas. Em outras palavras, não houve casos de setores censitários que começassem na terra indígena e terminassem fora delas. Esta característica facilitou sobremaneira os procedimentos analíticos aqui apresentados. No caso das terras indígenas em outras regiões do país, é possível que a sobreposição com os setores censitários do IBGE não seja tão concordante, o que gerará dificuldades importantes no cálculo de novos pesos para os procedimentos de expansão da amostra, tal como aqui realizado.

Para enfrentar essas dificuldades indicadas e criar condições para uma mais

\section{Referências}

AZEVEDO, M. M. Censos demográficos e "os índios": dificuldades para reconhecer e contar. In: RICARDO, C. A. (Org.). Povos indígenas no Brasil 1996/2000. São Paulo: Instituto Socioambiental, 2000, p. 79-83.

ALBIERI, S. Apresentação da precisão de estimativas nas tabelas de pesquisas por amostragem do IBGE. Rio de Janeiro: IBGE/DPE/COMEQ, 1999 (Relatório interno).

ALBIERI, S.; PEREIRA, N. O. M.; BRITO, J. A. M. População autodeclarada indígena: uma avaliação da precisão das estimativas nos Censos Demográficos de 1991 e 2000. In: XV ENCONTRO NACIONAL DE ESTUDOS POPULACIONAIS. Anais... Caxambu-MG, Abep, 18-22 de setembro de 2006.

ALBIERI, S. Mudança de escala na apresentação de estimativas pontuais: uma realidade. Rio de Janeiro: IBGE, 2008. Mimeografado.

BANKIER, M. D. Canadian Census sample Estimation Methodology. Presented at Interchange '90. Washington DC, 1990.

DIAS, A. R.; ALBIERI, S. As implicações do uso de calibração no procedimento de expansão da amostra do Censo Demográfico 2000. In: XIV ENCONTRO NACIONAL DE ESTUDOS POPULACIONAIS. Anais... CaxambuMG, Abep, 20-24 de setembro de 2004. fácil utilização dos dados censitários para os "indígenas", é importante que o próximo censo nacional, a ser realizado em 2010, conte com uma "base operacional" que represente, o mais satisfatoriamente possível, as terras indígenas. Denomina-se "base operacional" a composição, incluindo os limites, dos setores censitários, que constituem a unidade censitária. No que se refere aos "indígenas", seria muito importante que os setores censitários se sobrepusessem com as terras indígenas. Como visto, isso já acontece com as Terras Xavante, mas não é o caso de outras terras indígenas no país. Sanada esta dificuldade, seria mais fácil aplicar a metodologia aqui apresentada para os dados a serem derivados de futuros censos.

KARIYA, T.; KURATA, H. Generalized least squares. London: Wiley, 2004.

HIDIROGLOU, M. A. Structure of the Generalized Estimation System (GES). Business Survey Methods Division, 1991.

IBGE - Instituto Brasileiro de Geografia e Estatística. Metodologia do Censo Demográfico 2000. Rio de Janeiro, v. 25, Anexo 2.2, 2003 (Série Relatórios Metodológicos).

Tendências demográficas: uma análise dos indígenas com base nos resultados da amostra dos Censos Demográficos 1991 e 2000. Rio de Janeiro, 2005.

. Manual do recenseador-CD-
1.09 do Censo Demográfico 2000, p 15.
KENNEDY, D. P.; STEPHEN, G. P. Who
are Brazil's indígenas? Contributions of
census data analysis to anthropological
demography of indigenous populations.
Human Organization, 59(3): 311-324, 2000.
MCSWEENEY, K.; ARPS, S. A "demographic
turnaround": the rapid growth of the
indigenous populations in Lowland Latin
America. Latin American Research Review,
$40(1): 3-29,2005$.

PERZ, S. G.; JONATHAN, W.; KENNEDY, D. P. Contributions of racial-ethnic reclassification and demographic processes to indigenous 
population resurgence: the case of Brazil. Latin American Research Review, 43(2): 7-33, 2008.

PEREIRA, N. O. M.; SANTOS, R. V.; AZEVEDO, M. M. Perfil demográfico e socioeconômico das pessoas que se autodeclararam "indígenas" nos Censos Demográficos de 1991 e 2001. In: PAGLIARO, H.; AZEVEDO, M. M.; SANTOS, R. V. (Eds.). Demografia dos povos indígenas no Brasil. Rio de Janeiro: Editora Fiocruz/Associação Brasileira de Estudos Populacionais, 2005.

PEREIRA, N. O. M.; SANTOS, R. V.; WELCH, J. R.; SOUZA, L. G.; COIMBRA Jr., C. E. A. Demography, territory, and identity of indigenous peoples in Brazil: the Xavante indians and the 2000 brazilian national census. Human Organization, 68(2): 166180, 2009.

PAGLIARO, H.; AZEVEDO; M. M.; SANTOS, R. V. (Eds.). Demografia dos povos indígenas no Brasil. Rio de Janeiro: Editora Fiocruz/Associação Brasileira de Estudos Populacionais, 2005.

SILVA, P. L. do N.; BIANCHINI, Z. M.; ALBIERI, S. Uma proposta de metodologia para a expansão da amostra do censo demográfico de 1991. Rio de Janeiro: IBGE, 1993 (Textos para discussão, 62).

SILVA, A. N.; MATZENBACHER, L. A.; CORTEZ, B. F. Processamento das áreas de expansão e disseminação da amostra no censo demográfico 2000. Rio de Janeiro: IBGE, Diretoria de Pesquisas, Coordenação de Métodos e Qualidade, 2002.

\title{
Resumen
}

¿Cómo tratar los datos de la muestra del Censo Demográfico 2000 en la obtención de estimativas para los "indígenas"? Un estudio a partir de las Tierras Indígenas Xavante, Mato Grosso

La demografía de los pueblos indígenas en Brasil es aún muy poco conocida en sus más diversos aspectos. En los últimos años, se viene dando una ampliación de interés por el tema, con la publicación de diversos estudios sobre demografía de los "indígenas" con base en los dados censales. Una dificultad metodológica importante habla respecto de la expansión de la muestra de los censos, ya que las tierras indígenas no fueron definidas originalmente como áreas de ponderación. Este trabajo presenta estimativas para las variables del cuestionario de la "muestra", considerando un conjunto de sectores censales pertenecientes a tierras indígenas y utilizando como estudio de caso las Tierras Xavante localizadas en el este de Mato Grosso, constituidas por seis áreas no-contiguas. Se trata de un ejercicio metodológico que tiene como objetivo comparar y evaluar las estimativas producidas según los pesos generados en la época de divulgación del Censo 2000 y los nuevos pesos calculados a partir de la metodología aquí presentada. Desde el punto de vista metodológico, este procedimiento es innovador, pues puede ser útil para estimar, con base en los datos de la muestra del Censo 2000, características de áreas no-contiguas y diferentes de aquéllas definidas para la expansión de la muestra como fue originalmente realizada y divulgada por el IBGE.

Palabras-clave: Censos demográficos. Color o raza. Indígenas. Muestreo.

\begin{abstract}
How to treat data from the sample of the 2000 Demographic Census to obtain estimates for native peoples in Brazil? A study based on the Lands of the Xavante Indians in the State of Mato Grosso, Brazil
\end{abstract}

The demography of the Brazilian native peoples is still uncharted territory in its numerous and diversified aspects. There has been growing interest in the topic in recent years, with the 
publication of a number of studies on the demography of native peoples, on the basis of census data. One major methodological difficulty is the problem of expanding the census samples, since the lands of the native peoples were not originally defined as weighted areas. This article presents estimates for the variables on the questionnaire for the "sample," considering one set of census sectors belonging to the lands of native peoples, using as a case study the lands of the Xavante Indians, consisting of six non-contiguous areas located in the eastern part of the Brazilian State of Mato Grosso. This methodological exercise is designed to compare and evaluate the estimates produced according to the weights generated at the time of disclosure of the Census 2000 results and the new weights calculated on the basis of the methodology presented here. From the methodological point of view, this procedure is innovative as it might be useful for estimating, on the basis of the data from the sample on the 2000 Census, characteristics of areas that are non-contiguous and different from those defined for expanding the sample as originally conceived and published by the Brazilian Census Office.

Keywords: Demographic censuses. Color or ethnic group. Native peoples. Sample.

Recebido para publicação em 26/05/2009. Aceito para publicação em 02/09/2009. 
